\title{
Full Satisfiability of UML Class Diagrams
}

\author{
Alessandro Artale, Diego Calvanese, and Angélica Ibáñez-García \\ KRDB Research Centre, Free University of Bozen-Bolzano, Italy \\ \{artale, calvanese, ibanezgarcia\}@inf .unibz.it
}

\begin{abstract}
UML class diagrams (UCDs) are the de-facto standard formalism for the analysis and design of information systems. By adopting formal language techniques to capture constraints expressed by UCDs one can exploit automated reasoning tools to detect relevant properties, such as schema and class satisfiability and subsumption between classes. Among the reasoning tasks of interest, the basic one is detecting full satisfiability of a diagram, i.e., whether there exists an instantiation of the diagram where all classes and associations of the diagram are non-empty and all the constraints of the diagram are respected. In this paper we establish tight complexity results for full satisfiability for various fragments of UML class diagrams. This investigation shows that the full satisfiability problem is ExPTIME-complete in the full scenario, NP-complete if we drop ISA between relationships, and NLOGSPACE-complete if we further drop covering over classes 1
\end{abstract}

Keywords: Reasoning over Conceptual Models, Description Logics, Complexity Analysis.

\section{Introduction}

UML (Unified Modeling Language - http://www.omg.org/spec/UML/) is the de-facto standard formalism for the analysis and design of information systems. One of the most important components of UML are class diagrams (UCDs). UCDs describe the domain of interest in terms of objects organized in classes and associations between them. The semantics of UCDs is by now well established, and several works propose to represent it using various kinds of formal languages, e.g., [2 344567]. Thus, one can in principle reason on UCDs. The reasoning tasks that one is interested in are, e.g., subsumption between two classes, i.e., the fact that each instance of one class is necessarily also an instance of another class, satisfiability of a specific class (or association) in the diagram, i.e., the fact that the information encoding that class (or association) in the diagram is not contradictory, diagram satisfiability, which requires that at least one class in the diagram is satisfiable, and full satisfiability of the diagram [8, , i.e., the fact that there exists an instantiation of the diagram where all classes and associations of the diagram are non-empty.

\footnotetext{
${ }^{1}$ A preliminary and shortened version of this paper has been presented at the 2009 Int. Workshop on Logic in Databases (LID 2009), with informal proceedings printed as a technical report [1.
} 
The latter property is of importance since the presence of some unsatisfiable class or association actually means either that the diagram contains unnecessary information that should be removed, or that there is some modeling error that leads to the loss of satisfiability.

In this paper, we adopt the well established formalization of UCDs in terms of Description Logics (DLs). DLs [10] are decidable logics that are specifically tailored for capturing various forms of conceptual data models (cf. [11 12 13 14 15 16 5]), and they allow one to exploit state-of-the-art DL reasoners [17] to provide automated reasoning support over such data models.

The complexity of reasoning over UCDs has been addressed in [5] where it has been shown that in the presence of the standard UML/EER constructs, such as ISA, disjointness and covering between entities and associations, cardinality constraints (also called participation constraints) for associations, and multiplicity constraints for attributes makes checking class satisfiability and schema satisfiability ExPTIME-complete. This result has been strengthened in [6] to UCD $2^{2}$ with simple ISA between associations (and both disjointness and completeness constraints on class hierarchies only), where it was also shown that by dropping ISA between associations reasoning becomes NP-complete, and by further forbidding completeness in class hierarchies it drops to NLOGSPACE-complete.

The only works that addressed explicitly the complexity of full satisfiability of UCDs are 89, which include a classification of UCDs based on inconsistency triggers. Each inconsistency trigger is a pattern for recognizing possible inconsistencies of the diagram based on the interaction between different modelling constraints. [8,9] introduce various algorithms for checking full satisfiability of UCDs with different expressive power, together with an analysis of their computational complexity (i.e., upper bounds are provided). In particular, checking full satisfiability in the following scenarios is showed to be in:

1. ExPTIME, if the standard constructs are used;

2. NP, if ISA between associations and multiple and overwriting inheritance of attributes is dropped - i.e., each attribute has a fixed type;

3. P, if diagrams are further restricted by forbidding completeness constraints;

4. PSPACE (instead ofExpTIME), if standard constructs are uses (as in scenario (1) but types for attributes associated to sub-classes are sub-types of types for the respective attributes associated to super-classes;

5. NP and $\mathrm{P}$ in the scenarios 2 and 3 , respectively, if we further allow for attributes with types restricted as in 4 .

The main contributions of this paper can be summarised as follows:

- We show tight complexity results for checking full satisfiability proving that the problem is ExPTIME-complete in the standard scenario 1. NP-complete in the scenario 2 and NLoGSPACE-complete (instead of P) in the scenario 3 .

- We prove that full satisfiability in the scenario 4 is ExPTImE-hard, thus showing that the PSPACE algorithm presented in [89] must be incomplete.

${ }^{2}$ The results in [6] are formulated in terms of the Entity-Relationship model, but they also carry directly over to UML class diagrams. 
Our results build on the formalization of UCDs in terms of DLs given in [5]6]. In fact, our upper bounds for full satisfiability are an almost direct consequence of the corresponding upper bounds of the DL formalization. On the other hand, the obtained lower bounds for full satisfiability are more involved, and in some cases require a careful analysis of the corresponding proof for class satisfiability.

The rest of the paper is organized as follows. In Section 2, we briefly introduce the DL $\mathcal{A L C}$, on which we base our results, and show that full satisfiability in $\mathcal{A L C}$ is ExpTIME-complete. In Section 3, we recall the FOL semantics of UCDs. In Sections 4 and 5, we provide our results on full satisfiability for various variants of UCDs. Finally, in Section 6, we draw some conclusions.

\section{$2 \quad$ Full Satisfiability in the Description Logic $\mathcal{A L C}$}

We start by studying full satisfiability for the DL $\mathcal{A L C}$, one of the basic variants of DLs [10. The basic elements of $\mathcal{A L C}$ are atomic concepts and roles, denoted by $A$ and $P$, respectively. Complex concepts $C, D$ are defined as follows:

$$
C, D::=A|\neg C| C \sqcap D \mid \exists P . C
$$

The semantics of $\mathcal{A L C}$, as usual in DLs, is specified in terms of interpretations. An interpretation $\mathcal{I}=\left(\Delta^{\mathcal{I}},{ }^{\mathcal{I}}\right)$, with a non-empty domain $\Delta^{\mathcal{I}}$ and an interpretation function ${ }^{\mathcal{I}}$, assigns to each concept $C$ a subset $C^{\mathcal{I}}$ of $\Delta^{\mathcal{I}}$, and to each role name $P$ a binary relation $P^{\mathcal{I}}$ in $\Delta^{\mathcal{I}} \times \Delta^{\mathcal{I}}$ such that the following conditions are satisfied:

$$
\begin{aligned}
A^{\mathcal{I}} & \subseteq \Delta^{\mathcal{I}}, & & (C \sqcap D)^{\mathcal{I}}=C^{\mathcal{I}} \cap D^{\mathcal{I}}, \\
(\neg C)^{\mathcal{I}} & =\Delta^{\mathcal{I}} \backslash C^{\mathcal{I}}, & & (\exists P . C)^{\mathcal{I}}=\left\{a \in \Delta^{\mathcal{I}} \mid \exists b .(a, b) \in P^{\mathcal{I}} \wedge b \in C^{\mathcal{I}}\right\} .
\end{aligned}
$$

We use the standard abbreviations $C_{1} \sqcup C_{2}:=\neg\left(\neg C_{1} \sqcap \neg C_{2}\right)$, and $\forall P . C:=$ $\neg \exists P . \neg C$, with the corresponding semantics.

An $\mathcal{A L C}$ terminological box (TBox) $\mathcal{T}$ is a finite set of (concept inclusion) assertions of the form $C \sqsubseteq D$. An interpretation $\mathcal{I}$ satisfies an assertion of the form $C \sqsubseteq D$ if and only if $C^{\mathcal{I}} \subseteq D^{\mathcal{I}}$. A TBox $\mathcal{T}$ is satisfiable if there is an interpretation $\mathcal{I}$, called a model of $\mathcal{T}$, that satisfies every assertion in $\mathcal{T}$. A concept $C$ is satisfiable w.r.t. a TBox $\mathcal{T}$ if there is a model $\mathcal{I}$ of $\mathcal{T}$ such that $C^{\mathcal{I}} \neq \emptyset$. It can be shown that TBox satisfiability and concept satisfiability w.r.t. a TBox are reducible to each other in polynomial time. Moreover, reasoning w.r.t. $\mathcal{A L C}$ TBoxes is ExPTIME-complete (see e.g., [10]).

We now define the notion of full satisfiability of a TBox and show that for $\mathcal{A L C}$ it has the same complexity as classical satisfiability.

Definition 1 (TBox Full Satisfiability). An $\mathcal{A L C}$ TBox $\mathcal{T}$ is said to be fully satisfiable if there exists a model $\mathcal{I}$ of $\mathcal{T}$ such that $A^{\mathcal{I}} \neq \emptyset$, for every atomic concept $A$ in $\mathcal{T}$. We say that $\mathcal{I}$ is a full model of $\mathcal{T}$.

We first prove that full satisfiability in $\mathcal{A L C}$ is ExpTime-hard. 
Lemma 2. Concept satisfiability w.r.t. $\mathcal{A L C}$ TBoxes can be linearly reduced to full satisfiability of $\mathcal{A L C}$ TBoxes.

Proof. Let $\mathcal{T}$ be an $\mathcal{A L C}$ TBox and $C$ an $\mathcal{A L C}$ concept. As pointed out in [18, $C$ is satisfiable w.r.t. $\mathcal{T}$ if and only if $C \sqcap A_{\mathcal{T}}$ is satisfiable w.r.t. the TBox $\mathcal{T}_{1}$ consisting of the single assertion, $A_{\mathcal{T}} \sqsubseteq \prod_{C_{1} \sqsubseteq C_{2} \in \mathcal{T}}\left(\neg C_{1} \sqcup C_{2}\right) \sqcap \prod_{1 \leq i \leq n} \forall P_{i} . A_{\mathcal{T}}$, where $A_{\mathcal{T}}$ is a fresh atomic concept and $P_{1}, \ldots, P_{n}$ are all the atomic roles in $\mathcal{T}$ and $C$. In order to reduce the latter problem to full satisfiability, we extend $\mathcal{T}_{1}$ to $\mathcal{T}_{2}=\mathcal{T}_{1} \cup\left\{A_{C} \sqsubseteq C \sqcap A_{\mathcal{T}}\right\}$, with $A_{C}$ a fresh atomic concept, and prove that: $C \sqcap A_{\mathcal{T}}$ is satisfiable w.r.t. $\mathcal{T}_{1}$ if and only if $\mathcal{T}_{2}$ is fully satisfiable.

" $\Rightarrow$ " Let $\mathcal{I}$ be a model of $\mathcal{T}_{1}$ such that $\left(C \sqcap A_{\mathcal{T}}\right)^{\mathcal{I}} \neq \emptyset$. We construct an interpretation of $\mathcal{T}_{2}, \mathcal{J}=\left(\Delta^{\mathcal{I}} \cup\left\{d^{\text {top }}\right\},{ }^{\mathcal{J}}\right)$, with $d^{\text {top }} \notin \Delta^{\mathcal{I}}$, such that:

$$
\begin{aligned}
& A_{\mathcal{T}}^{\mathcal{J}}=A_{\mathcal{T}}^{\mathcal{I}}, \quad A_{C}^{\mathcal{J}}=\left(C \sqcap A_{\mathcal{T}}\right)^{\mathcal{I}}, \\
& A^{\mathcal{J}}=A^{\mathcal{I}} \cup\left\{d^{\text {top }}\right\}, \quad \text { for each atomic concept } A \text { in } \mathcal{T} \text { and } C, \\
& P^{\mathcal{J}}=P^{\mathcal{I}}, \quad \text { for each atomic role } P \text { in } \mathcal{T} \text { and } C .
\end{aligned}
$$

Obviously, the extension of every atomic concept is non-empty in $\mathcal{J}$. Next, we show that $\mathcal{J}$ is a model of $\mathcal{T}_{2}$, by relying on the fact (easily proved by structural induction) that $D^{\mathcal{I}} \subseteq D^{\mathcal{J}}$, for each subconcept $D$ of concepts in $\mathcal{T}_{1}$ or of $C$. Then, it is easy to show that $\mathcal{J}$ satisfies the two assertion in $\mathcal{T}_{2}$.

$" \Leftarrow "$ Conversely, every full model $\mathcal{J}$ of $\mathcal{T}_{2}$ is also a model of $\mathcal{T}_{1}$ with $\left(C \sqcap A_{\mathcal{T}}\right)^{\mathcal{J}} \neq$ $\emptyset$, as $A_{C}^{\mathcal{J}} \subseteq\left(C \sqcap A_{\mathcal{T}}\right)^{\mathcal{J}}$.

Theorem 3. Full satisfiability of $\mathcal{A L C}$ TBoxes is ExpTImE-complete.

Proof. The ExpTime membership is straightforward since full satisfiability of an $\mathcal{A L C}$ TBox $\mathcal{T}$ can be reduced to satisfiability of the TBox $\mathcal{T} \cup \bigcup_{1 \leq i \leq n}\{\top \sqsubseteq$ $\left.\exists P^{\prime} . A_{i}\right\}$, where $A_{1}, \ldots, A_{n}$ are all the atomic concepts in $\mathcal{T}$, and $P^{\prime}$ is a fresh atomic role. The ExPTime-hardness follows from Lemma 2 ,

We now modify the reduction of Lemma 2 so that it applies also to primitive $\mathcal{A L C}^{-}$TBoxes, i.e., TBoxes that contain only assertions of the form:

$$
A \sqsubseteq B, \quad A \sqsubseteq \neg B, \quad A \sqsubseteq B \sqcup B^{\prime}, \quad A \sqsubseteq \forall P . B, \quad A \sqsubseteq \exists P . B,
$$

where $A, B, B^{\prime}$ are atomic concepts, and $P$ is an atomic role.

Theorem 4. Full satisfiability of primitive $\mathcal{A L C}^{-}$TBoxes is ExPTIMEcomplete.

Proof. The ExpTime membership follows from Theorem 3 . For proving the ExPTIME-hardness, we use a result in [5] showing that concept satisfiability in $\mathcal{A L C}$ can be reduced to atomic concept satisfiability w.r.t. primitive $\mathcal{A} \mathcal{L C}^{-}$ TBoxes. Let $\mathcal{T}^{-}=\left\{A_{j} \sqsubseteq D_{j} \mid 1 \leq j \leq m\right\}$ be a primitive $\mathcal{A L C} \mathcal{C}^{-}$TBox, and $A_{0}$ an atomic concept. By the proof of Lemma 2, we have that $A_{0}$ is satisfiable w.r.t. $\mathcal{T}^{-}$if and only if the TBox $\mathcal{T}_{2}^{\prime}$ consisting of the assertions

$$
A_{\mathcal{T}^{-}} \sqsubseteq \prod_{A_{j} \sqsubseteq D_{j} \in \mathcal{T}^{-}}\left(\neg A_{j} \sqcup D_{j}\right) \sqcap \prod_{1 \leq i \leq n} \forall P_{i} \cdot A_{\mathcal{T}^{-}}, \quad \quad A_{0}^{\prime} \sqsubseteq A_{0} \sqcap A_{\mathcal{T}^{-}},
$$


is fully satisfiable, with $A_{\mathcal{T}^{-}}, A_{0}^{\prime}$ fresh atomic concepts.

$\mathcal{T}_{2}^{\prime}$ is not a primitive $\mathcal{A L C} \mathcal{C}^{-}$TBox, but it is equivalent to the TBox containing the assertions:

$$
\begin{array}{lcc}
A_{0}^{\prime} \sqsubseteq A_{\mathcal{T}^{-}} & A_{\mathcal{T}^{-}} \sqsubseteq \neg A_{1} \sqcup D_{1} & A_{\mathcal{T}^{-}} \sqsubseteq \forall P_{1} \cdot A_{\mathcal{T}^{-}} \\
& \vdots & \vdots \\
A_{0}^{\prime} \sqsubseteq A_{0} & A_{\mathcal{T}^{-}} \sqsubseteq \neg A_{m} \sqcup D_{m} & A_{\mathcal{T}^{-}} \sqsubseteq \forall P_{n} . A_{\mathcal{T}^{-}},
\end{array}
$$

Finally, to get a primitive $\mathcal{A L C}{ }^{-}$TBox, $\mathcal{T}_{2}^{-}$, we replace each assertion of the form $A_{\mathcal{T}^{-}} \sqsubseteq \neg A_{j} \sqcup D_{j}$ by $A_{\mathcal{T}^{-}} \sqsubseteq B_{j}^{1} \sqcup B_{j}^{2}, B_{j}^{1} \sqsubseteq \neg A_{j}$, and $B_{j}^{2} \sqsubseteq D_{j}$, with $B_{j}^{1}$ and $B_{j}^{2}$ fresh atomic concepts, for $j \in\{1, \ldots, m\}$.

We show now that $\mathcal{T}_{2}^{\prime}$ is fully satisfiable iff $\mathcal{T}_{2}^{-}$is fully satisfiable:

$(\Rightarrow)$ Let $\mathcal{I}=\left(\Delta^{\mathcal{I}},{ }^{\mathcal{I}}\right)$ be a full model of $\mathcal{T}_{2}^{\prime}$. We extend $\mathcal{I}$ to an interpretation $\mathcal{J}$ of $\mathcal{T}_{2}^{-}$. Let $\Delta^{\mathcal{J}}=\Delta^{\mathcal{I}} \cup\left\{d^{+}, d^{-}\right\}$, with $\left\{d^{+}, d^{-}\right\} \cap \Delta^{\mathcal{I}}=\emptyset$, and define ${ }^{\mathcal{J}}$ as follows:

$$
\begin{aligned}
A_{\mathcal{T}^{-}}^{\mathcal{J}} & =A_{\mathcal{T}^{-}}^{\mathcal{I}}, \quad A_{0}^{\prime \mathcal{J}}=A_{0}^{\prime \mathcal{I}}, \\
A^{\mathcal{J}} & =A^{\mathcal{I}} \cup\left\{d^{+}\right\}, \quad \text { for every other atomic concept } A \text { in } \mathcal{T}_{2}^{\prime}, \\
B_{j}^{1}{ }^{\mathcal{J}} & =\left(\neg A_{j}\right)^{\mathcal{J}} \text { and } B_{j}^{2}{ }^{\mathcal{J}}=D_{j}^{\mathcal{J}}, \quad \text { for each } A_{\mathcal{T}^{-}} \sqsubseteq B_{j}^{1} \sqcup B_{j}^{2} \in \mathcal{T}_{2}^{-}, \\
P^{\mathcal{J}} & =P^{\mathcal{I}} \cup\left\{\left(d^{+}, d^{+}\right)\right\}, \quad \text { for each atomic role } P \text { in } \mathcal{T}_{2}^{-} .
\end{aligned}
$$

It is easy to see that $\mathcal{J}$ is a full model of $\mathcal{T}_{2}^{-}$.

$(\Leftarrow)$ Trivial, since every model of $\mathcal{T}_{2}^{-}$is a model of $\mathcal{T}_{2}^{\prime}$.

\section{Formalizing UML Class Diagrams}

In this section, we briefly describe UCDs and provide their semantics in terms of First Order Logic (the formalization adopted here is based on previous presentations in $[515])$.

A class in UCDs denotes a set of objects with common features. Formally, a class $C$ corresponds to a unary predicate $C$. An $n$-ary association (also called relationship) in UCDs represents a relation between instances $n \geq 2$ classes. Names of associations (as names of classes) are unique in a UCD. A binary association between two classes $C_{1}$ and $C_{2}$ is graphically rendered as in Fig. 1 . The multiplicity constraint $n_{l} . . n_{u}$ (also called participation constraint) written on one end of the binary association specifies that each instance of the class $C_{1}$ participates at least $n_{l}$ times and at most $n_{u}$ times in the association $R$, and the multiplicity constraint $m_{l} . . m_{u}$ specifies an analogous constraint for each instance of the class $C_{2}$. When a multiplicity constraint is omitted, it is intended to be $0 . . *$. Formally, an association $R$ between the classes $C_{1}$ and $C_{2}$ is captured by a binary predicate $R$ that satisfies the FOL axiom $\forall x_{1}, x_{2} \cdot\left(R\left(x_{1}, x_{2}\right) \rightarrow C_{1}\left(x_{1}\right) \wedge C_{2}\left(x_{2}\right)\right)$, while multiplicities are formalized by the following FOL assertions:

$$
\begin{aligned}
& \forall x \cdot\left(C_{1}(x) \rightarrow \exists_{\geq n_{l}} y \cdot R(x, y) \wedge \exists_{\leq n_{u}} y \cdot R(x, y)\right) \\
& \forall y .\left(C_{2}(y) \rightarrow \exists_{\geq m_{l}} x \cdot R(x, y) \wedge \exists_{\leq m_{u}} x \cdot R(x, y)\right),
\end{aligned}
$$




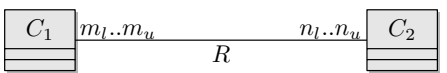

Fig. 1. Binary association

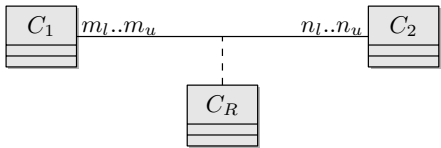

Fig. 2. Binary association with related class

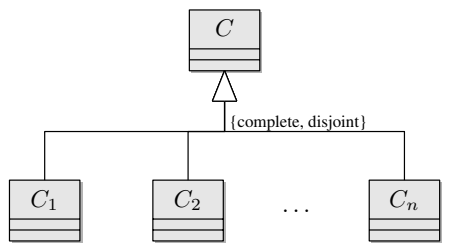

Fig. 3. A class hierarchy in UML

where we use counting quantifiers to abbreviate the FOL formula encoding the multiplicity constraints.

A more general form of multiplicity is the so called refinement of multiplicity constraints for sub-classes participating in associations. With such a construct we are able to change (and thus refine) the multiplicity constraints for sub-classes. Refinement involving a binary association, $R$, between classes $C_{1}$ and $C_{2}$, and a sub-class of $C_{1}$, say $C_{1}^{\prime}$, can be formalized with the following FOL axioms:

$$
\forall x .\left(C_{1}^{\prime}(x) \rightarrow C_{1}(x)\right), \quad \forall x .\left(C_{1}^{\prime}(x) \rightarrow \exists_{\geq n_{l}^{\prime}} y \cdot R(x, y) \wedge \exists_{\leq n_{u}^{\prime}} y \cdot R(x, y)\right) .
$$

An association class describes properties of the association, such as attributes, operations, etc. (see Fig. 2). A binary association between classes $C_{1}$ and $C_{2}$ with a related association class $C_{R}$ is formalized in FOL by reifying the association into a unary predicate $C_{R}$ with two binary predicates $P_{1}, P_{2}$, one for each component of the association. We enforce the following semantics, for $i \in\{1,2\}$ :

$$
\begin{aligned}
\forall x \cdot\left(C_{R}(x)\right. & \left.\rightarrow \exists y \cdot P_{i}(x, y)\right), \\
\forall x, y \cdot\left(C_{R}(x)\right. & \left.\wedge P_{i}(x, y) \rightarrow C_{i}(y)\right), \\
\forall x, y, y^{\prime} \cdot\left(C_{R}(x)\right. & \left.\wedge P_{i}(x, y) \wedge P_{i}\left(x, y^{\prime}\right) \rightarrow y=y^{\prime}\right), \\
\forall y_{1}, y_{2}, x, x^{\prime} \cdot\left(C_{R}(x)\right. & \left.\wedge C_{R}\left(x^{\prime}\right) \wedge\left(\bigwedge_{i \in\{1,2\}} P_{i}\left(x, y_{i}\right) \wedge P_{i}\left(x^{\prime}, y_{i}\right)\right) \rightarrow x=x^{\prime}\right) .
\end{aligned}
$$

For associations with a related class, the multiplicity constraints are formalized by the following FOL assertions:

$$
\begin{aligned}
& \forall y_{1} \cdot\left(C_{1}\left(y_{1}\right) \rightarrow \exists_{\geq n_{l}} x .\left(C_{R}(x) \wedge P_{1}\left(x, y_{1}\right)\right) \wedge \exists_{\leq n_{u}} x .\left(C_{R}(x) \wedge P_{1}\left(x, y_{1}\right)\right)\right), \\
& \forall y_{2} \cdot\left(C_{2}\left(y_{2}\right) \rightarrow \exists_{\geq m_{l}} x .\left(C_{R}(x) \wedge P_{2}\left(x, y_{2}\right)\right) \wedge \exists_{\leq m_{u}} x .\left(C_{R}(x) \wedge P_{2}\left(x, y_{2}\right)\right)\right) .
\end{aligned}
$$

Classes can have attributes, formalized similarly to binary associations, relating the class with values of a given type. As for associations, we can specify multiplicity constraints over attributes.

A generalization (called also ISA constraint) between two classes $C_{1}$ and $C$, formalized as $\forall x . C_{1}(x) \rightarrow C(x)$, specifies that each instance of $C_{1}$ is also an 
Table 1. Complexity of Full Satisfiability in UML (sub)languages

\begin{tabular}{|c|c|c|c|c|c|}
\hline \multirow{3}{*}{ Language } & \multicolumn{4}{|c|}{ Constraints } & \multirow{3}{*}{$\begin{array}{l}\text { Complexity } \\
\text { of Full } \\
\text { Satisfiability }\end{array}$} \\
\hline & \multicolumn{2}{|c|}{ Classes } & \multicolumn{2}{|c|}{ Associations/Attributes } & \\
\hline & ISA disjol & mplete & ISA multipl & nement & \\
\hline$\overline{\mathrm{UCD}_{\text {full }}}$ & $\begin{array}{ll}\checkmark & \checkmark\end{array}$ & $\checkmark$ & $\begin{array}{ll} & \checkmark\end{array}$ & $\checkmark$ & ExPTime [Th7] \\
\hline $\mathrm{UCD}_{b o o l}$ & $\checkmark$ & $\checkmark$ & $\checkmark$ & $\checkmark$ & $\mathrm{NP}$ [Th9] \\
\hline $\mathrm{UCD}_{r e f}$ & $\checkmark$ & $x$ & $\checkmark$ & $\checkmark$ & NLOGSpaCe [Th 11$]$ \\
\hline
\end{tabular}

instance of $C$. Several generalizations can be grouped together to form a class hierarchy, as shown in Fig. 3. Such a hierarchy is formally captured by means of the FOL axioms $\forall x . C_{i}(x) \rightarrow C(x)$ for $i \in\{1, \ldots, n\}$. Disjointness and completeness constraints can also be enforced on a class hierarchy, by adding suitable labels to the diagram. Disjointness among the classes $C_{1}, \ldots, C_{n}$ is expressed by $\forall x . C_{i}(x) \rightarrow \bigwedge_{j=i+1}^{n} \neg C_{j}(x)$, for $i \in\{1, \ldots, n-1\}$. The completeness constraint, expressing that each instance of $C$ is an instance of at least one of $C_{1}, \ldots, C_{n}$, is captured by $\forall x . C(x) \rightarrow \bigvee_{i=1}^{n} C_{i}(x)$. We can also have generalization, disjointness and completeness constraints between associations and between association classes with the obvious semantics.

In this paper, we denote with $\mathrm{UCD}_{\text {full }}$ the class diagram language that comprises all the standard constructs as discussed above (i.e., what we called scenario 1 in Section 1). With $\mathrm{UCD}_{b o o l}$ we denote the language without generalization between associations (i.e., scenario 2 in Section 1), and with $\mathrm{UCD}_{\text {ref }}$ we further drop completeness constraints over classes (i.e., scenario 3 in Section 1). The constructors allowed in these languages are summarized in Table 1 together with the tight complexity results obtained in this paper.

\section{Full Satisfiability of UML Class Diagrams}

Three notions of UCD satisfiability have been proposed in the literature [1956209]. First, diagram satisfiability refers to the existence of a model, i.e., a FOL interpretation that satisfies all the FOL assertions associated to the diagram and where at least one class has a nonempty extension. Second, class satisfiability refers to the existence of a model of the diagram where the given class has a nonempty extension. Third, we can check whether there is a model of an UML diagram that satisfies all classes and all relationships in a diagram. This last notion of satisfiability, referred here as full satisfiability and introduced in 89. is thus stronger than diagram satisfiability, since a model of a diagram that satisfies all classes is, by definition, also a model of that diagram.

Definition 5 (UML Full Satisfiability). A UCD, D, is fully satisfiable if there is a FOL interpretation, $\mathcal{I}$, that satisfies all the constraints expressed in $\mathcal{D}$ and such that $C^{\mathcal{I}} \neq \emptyset$ for every class $C$ in $\mathcal{D}$, and $R^{\mathcal{I}} \neq \emptyset$ for every association $R$ in $\mathcal{D}$. We say that $\mathcal{I}$ is a full model of $\mathcal{D}$. 


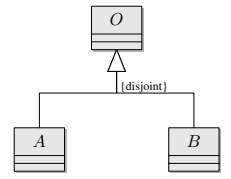

Fig. 4. Encoding of $A \sqsubseteq \neg B$

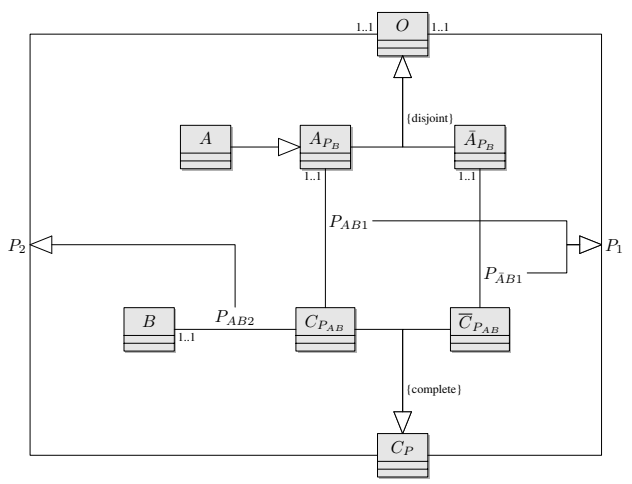

Fig. 6. Encoding of $A \sqsubseteq \forall P . B$

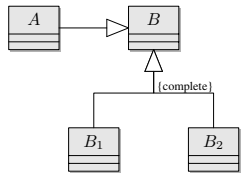

Fig. 5. Encoding of $A \sqsubseteq B_{1} \sqcup B_{2}$

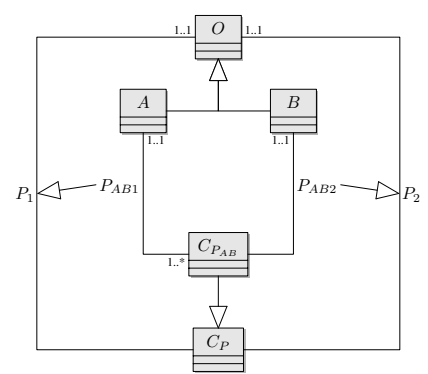

Fig. 7. Encoding of $A \sqsubseteq \exists P . B$

We now address the complexity of full satisfiability for UCDs with the standard set of constructs, i.e., $\mathrm{UCD}_{\text {full }}$. For the lower bounds, we use the results presented in Section 2 and reduce full satisfiability of primitive $\mathcal{A L C}{ }^{-}$TBoxes to full satisfiability of $\mathrm{UCD}_{\text {full }}$. This reduction is based on the ones used in $[56]$ for the lower complexity bound of schema satisfiability in the extended EntityRelationship model, but the proof of their correctness is more involved here.

Given a primitive $\mathcal{A L C} \mathcal{C}^{-}$TBox $\mathcal{T}$, construct a $\operatorname{UCD}_{\text {full }}$ diagram $\Sigma(\mathcal{T})$ as follows: for each atomic concept $A$ in $\mathcal{T}$, introduce a class $A$ in $\Sigma(\mathcal{T})$. Additionally, introduce a class $O$ that generalizes (possibly indirectly) all the classes in $\Sigma(\mathcal{T})$ that encode an atomic concept in $\mathcal{T}$. For each atomic role $P$, introduce a class $C_{P}$, which reifies the binary relation $P$. Further, introduce two functional associations $P_{1}$, and $P_{2}$ that represent, respectively, the first and second component of $P$. The assertions in $\mathcal{T}$ are encoded as follows:

- For each assertion of the form $A \sqsubseteq B$, introduce a generalization between the classes $A$ and $B$.

- For each assertion of the form $A \sqsubseteq \neg B$, construct the hierarchy in Fig. 4 .

- For each assertion of the form $A \sqsubseteq B_{1} \sqcup B_{2}$, introduce an auxiliary class $B$, and construct the diagram shown in in Fig. 5 .

- For each assertion of the form $A \sqsubseteq \forall P . B$, add the auxiliary classes $C_{P_{A B}}$, $\bar{C}_{P_{A B}}, A_{P_{B}}$, and $\bar{A}_{P_{B}}$, and the associations $P_{A B 1}, P_{\bar{A} B 1}$, and $P_{A B 2}$, and construct the diagram shown in Fig. 6 . 
- For each assertion of the form $A \sqsubseteq \exists P . B$, add the auxiliary class $C_{P_{A B}}$ and the associations $P_{A B 1}$ and $P_{A B 2}$, and construct the diagram shown in Fig. 7

Notice that $\Sigma(\mathcal{T})$ is a UCD in $\mathrm{UCD}_{\text {full }}$.

Lemma 6. A primitive $\mathcal{A L C} \mathcal{C}^{-}$TBox $\mathcal{T}$ is fully satisfiable if and only if the $U C D$ $\Sigma(\mathcal{T})$, constructed as above, is fully satisfiable.

Proof. " $\Leftarrow "$ Let $\mathcal{J}=\left(\Delta^{\mathcal{J}}, \cdot^{\mathcal{J}}\right)$ be a full model of $\Sigma(\mathcal{T})$. We construct a full model $\mathcal{I}=\left(\Delta^{\mathcal{I}},{ }^{\mathcal{I}}\right)$ of $\mathcal{T}$ by taking $\Delta^{\mathcal{I}}=\Delta^{\mathcal{J}}$. Further, for every concept name $A$ and for every atomic role $P$ in $\mathcal{T}$, we define respectively $A^{\mathcal{I}}=A^{\mathcal{J}}$ and $P^{\mathcal{I}}=\left(P_{1}^{-}\right)^{\mathcal{J}} \circ P_{2}^{\mathcal{J}}\left(r_{1} \circ r_{2}\right.$ denotes the composition of two binary relations $r_{1}$ and $\left.r_{2}\right)$. Let us show that $\mathcal{I}$ satisfies every assertion in $\mathcal{T}$.

- For assertions of the form $A \sqsubseteq B, A \sqsubseteq \neg B$, and $A \sqsubseteq B_{1} \sqcup B_{2}$, the statement easily follows from the construction of $\mathcal{I}$.

- For assertions of the form $A \sqsubseteq \forall P . B$ and $A \sqsubseteq \exists P . B$, the proof uses arguments similar to those in the proof of Lemma 1 in $[6]$.

" $\Rightarrow$ " Let $\mathcal{I}=\left(\Delta^{\mathcal{I}},{ }^{\mathcal{I}}\right)$ be a full model of $\mathcal{T}$, and let $\operatorname{role}(\mathcal{T})$ be the set of role names in $\mathcal{T}$. We extend $\mathcal{I}$ to an instantiation $\mathcal{J}=\left(\Delta^{\mathcal{J}},{ }^{\mathcal{J}}\right)$ of $\Sigma(\mathcal{T})$, by assigning suitable extensions to the auxiliary classes and associations in $\Sigma(\mathcal{T})$. Let $\Delta^{\mathcal{J}}=\Delta^{\mathcal{I}} \cup \Gamma \cup \Lambda$, where: $\Lambda=\biguplus_{A \sqsubseteq \forall P . B \in \mathcal{T}}\left\{a_{A_{P_{B}}}, a_{\bar{A}_{P_{B}}}\right\}$, such that $\Delta^{\mathcal{I}} \cap \Lambda=$ $\emptyset$, and $\Gamma=\biguplus_{P \in \operatorname{role}(\mathcal{T})} \Delta_{P}$, with $\Delta_{P}=P^{\mathcal{I}} \cup \bigcup_{A \sqsubseteq \forall P . B \in \mathcal{T}}\left\{\left(a_{A_{P_{B}}}, b\right),\left(a_{\bar{A}_{P_{B}}}, \bar{o}\right)\right\}$ where $b$ is an arbitrary instance of $B$, and $\bar{o}$ an arbitrary element of $\Delta^{\mathcal{I}}$. We set $O^{\mathcal{J}}=\Delta^{\mathcal{I}} \cup \Lambda, A^{\mathcal{J}}=A^{\mathcal{I}}$ for each class $A$ corresponding to an atomic concept in $\mathcal{T}$, and $C_{P}^{\mathcal{J}}=\Delta_{P}$ for each $P \in \operatorname{role}(\mathcal{T})$. Additionally, the extensions of the associations $P_{1}$ and $P_{2}$ are defined as follows: $P_{1}^{\mathcal{J}}=\left\{\left(\left(o_{1}, o_{2}\right), o_{1}\right) \mid\left(o_{1}, o_{2}\right) \in\right.$ $\left.C_{P}^{\mathcal{J}}\right\}, \quad P_{2}^{\mathcal{J}}=\left\{\left(\left(o_{1}, o_{2}\right), o_{2}\right) \mid\left(o_{1}, o_{2}\right) \in C_{P}^{\mathcal{J}}\right\}$. We now show that $\mathcal{J}$ is a full model of $\Sigma(\mathcal{T})$.

- For the portions of $\Sigma(\mathcal{T})$ due to TBox assertions of the form $A \sqsubseteq B, A \sqsubseteq \neg B$, and $A \sqsubseteq B_{1} \sqcup B_{2}$, the statement follows from the construction of $\mathcal{J}$.

- For each TBox assertion in $\mathcal{T}$ of the form $A \sqsubseteq \forall P . B$, let us define the extensions for the auxiliary classes and associations as follows:

$A_{P_{B}}^{\mathcal{J}}=A^{\mathcal{I}} \cup\left\{a_{A_{P_{B}}}\right\}$,

$\bar{A}_{P_{B}}^{\mathcal{J}}=O^{\mathcal{J}} \backslash A_{P_{B}}^{\mathcal{J}}$,

$C_{P_{A B}}^{\mathcal{J}}=\left\{\left(o, o^{\prime}\right) \in C_{P}^{\mathcal{J}} \mid o \in A_{P_{B}}^{\mathcal{J}}\right\}$,

$\bar{C}_{P_{A B}}^{\mathcal{J}}=\left\{\left(o, o^{\prime}\right) \in C_{P}^{\mathcal{J}} \mid o \in \bar{A}_{P_{B}}^{\mathcal{J}}\right\}$,

$P_{A B 1}^{\mathcal{J}}=\left\{\left(\left(o, o^{\prime}\right), o\right) \in P_{1}^{\mathcal{J}} \mid o \in A_{P_{B}}^{\mathcal{J}}\right\}$,

$P_{\bar{A} B 1}^{\mathcal{J}}=\left\{\left(\left(o, o^{\prime}\right), o\right) \in P_{1}^{\mathcal{J}} \mid o \in \bar{A}_{P_{B}}^{\mathcal{J}}\right\}$

$P_{A B 2}^{\mathcal{J}}=\left\{\left(\left(o, o^{\prime}\right), o^{\prime}\right) \in P_{2}^{\mathcal{J}} \mid o \in A_{P_{B}}^{\mathcal{J}}\right\}$.

It is not difficult to see that $\mathcal{J}$ satisfies the fragment of $\Sigma(\mathcal{T})$ as shown in Fig. 6. It remains to show that each class and each association has a non-empty extension. This is clearly the case for classes that encode atomic concepts in $\mathcal{T}$. For the classes $A_{P_{B}}, \bar{A}_{P_{B}}, C_{P_{A B}}$, and $\bar{C}_{P_{A B}}$ we have that

$$
a_{A_{P_{B}}} \in A_{P_{B}}^{\mathcal{J}}, \quad a_{\bar{A}_{P_{B}}} \in \bar{A}_{P_{B}}^{\mathcal{J}}, \quad\left(a_{A_{P_{B}}}, b\right) \in C_{P_{A B}}^{\mathcal{J}}, \quad\left(a_{\bar{A}_{P_{B}}}, \bar{o}\right) \in \bar{C}_{P_{A B}}^{\mathcal{J}} .
$$



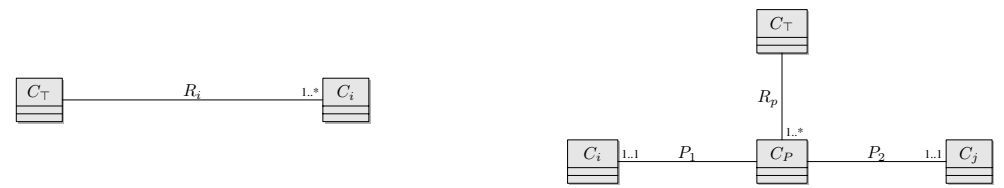

Fig. 8. Reducing UML full satisfiability to class satisfiability

For the associations $P_{1}, P_{2}, P_{A B 1}, P_{A B 2}$, and $P_{\bar{A} B 1}$ we have that $\left(\left(a_{A_{P_{B}}}, b\right), a_{A_{P_{B}}}\right) \in P_{A B 1}^{\mathcal{J}} \subseteq P_{1}^{\mathcal{J}}, \quad\left(\left(a_{\bar{A}_{P_{B}}}, \bar{o}\right), a_{\bar{A}_{P_{B}}}\right) \in P_{\bar{A} B 1}^{\mathcal{J}}, \quad\left(\left(a_{A_{P_{B}}}, b\right), b\right) \in$ $P_{A B 2}^{\mathcal{J}} \subseteq P_{2}^{\mathcal{J}}$.

- For each TBox assertion in $\mathcal{T}$ of the form $A \sqsubseteq \exists P$. B , let us define:

$$
\begin{aligned}
C_{P_{A B}}^{\mathcal{J}} & =\left\{\left(o, o^{\prime}\right) \in C_{P}^{\mathcal{J}} \mid o \in A^{\mathcal{I}} \text { and } o^{\prime} \in B^{\overline{\mathcal{I}}}\right\}, \\
P_{A B 1}^{\mathcal{J}} & =\left\{\left(\left(o, o^{\prime}\right), o\right) \in P_{1}^{\mathcal{J}} \mid\left(o, o^{\prime}\right) \in C_{P_{A B}}^{\mathcal{J}}\right\}, \\
P_{A B 2}^{\mathcal{J}} & =\left\{\left(\left(o, o^{\prime}\right), o^{\prime}\right) \in P_{2}^{\mathcal{J}} \mid\left(o, o^{\prime}\right) \in C_{P_{A B}}^{\mathcal{J}}\right\} .
\end{aligned}
$$

We have that $C_{P_{A B}}^{\mathcal{J}} \neq \emptyset$ as there exists a pair $(a, b) \in \Delta_{P}$ with $a \in A^{\mathcal{I}}$, and $b \in B^{\mathcal{I}}$. Since $C_{P_{A B}}^{\mathcal{J}} \neq \emptyset$, we have that $P_{A B 1}^{\mathcal{J}} \neq \emptyset$ and $P_{A B 2}^{\mathcal{J}} \neq \emptyset$.

Theorem 7. Full satisfiability of $U C D_{\text {full }}$ diagrams is ExPTIME-complete.

Proof. We establish the upper bound by a reduction to class satisfiability in UCDs, which is known to be ExPTImE-complete [5]. Given a UCD $\mathcal{D}$, with classes $C_{1}, \ldots, C_{n}$, we construct a UCD $\mathcal{D}^{\prime}$ by adding to $\mathcal{D}$ a new class $C_{\top}$ and new associations $R_{i}$, for $i \in\{1, \ldots, n\}$, as shown in the left part of Fig. 8 , Furthermore, to check that every association is populated we use reification, i.e., we replace each association $P$ in the diagram $\mathcal{D}$ between the classes $C_{i}$ and $C_{j}$ (such that neither $C_{i}$ nor $C_{j}$ is constrained to participate at least once to $P$ ) with a class $C_{P}$ and two functional associations $P_{1}$ and $P_{2}$ to represent each component of $P$. Finally, we add the constraints shown in the right part of Fig. 8. Intuitively, we have that if there is a model $\mathcal{I}$ of the extended diagram $\mathcal{D}^{\prime}$ in which $C_{\top}^{\mathcal{I}} \neq \emptyset$, then the multiplicity constraint $1 . . *$ on the association $R_{P}$ forces the existence of at least one instance $o$ of $C_{P}$. By the functionality of $P_{1}$ and $P_{2}$ there are at least two elements $o_{i}$ and $o_{j}$, such that $o_{i} \in C_{i}^{\mathcal{I}}, o_{j} \in C_{j}^{\mathcal{I}}$, $\left(o, o_{i}\right) \in P_{1}^{\mathcal{I}}$ and $\left(o, o_{j}\right) \in P_{2}^{\mathcal{I}}$. Then, one instance of $P$ can be the pair $\left(o_{i}, o_{j}\right)$. Conversely, if there is a full model $\mathcal{J}$ of $\mathcal{D}$, it is easy to extend it to a model $\mathcal{I}$ of $\mathcal{D}^{\prime}$ that satisfies $C_{\top}$.

The ExpTime-hardness follows from Lemma 6 and Theorem 4

Note that, the proof of the above theorem does not involve attributes. Thus, the ExPTImE complexity result is valid for both scenarios 1 and 4 in Section 1.

\section{Full Satisfiability of Restricted UML Class Diagrams}

In this section, we investigate the complexity of the full satisfiability problem for the two sub-languages $\mathrm{UCD}_{b o o l}$ and $\mathrm{UCD}_{\text {ref }}$ defined in Section 3 , By building 
on the techniques used for the satisfiability proofs in [6], we show that also in this case checking for full satisfiability does not change the complexity of the problem.

We consider first $\mathrm{UCD}_{\text {bool }}$ diagrams, by showing that deciding full satisfiability is NP-complete. For the lower bound, we provide a polynomial reduction of the 3SAT problem (which is known to be NP-complete) to full satisfiability of $\mathrm{UCD}_{\text {bool }}$ CDs.

Let an instance of 3SAT be given by a set $\phi=\left\{c_{1}, \ldots, c_{m}\right\}$ of 3-clauses over a finite set $\Pi$ of propositional variables. Each clause is such that $c_{i}=\ell_{i}^{1} \vee \ell_{i}^{2} \vee \ell_{i}^{3}$, for $i \in\{1, \ldots, m\}$, where each $\ell_{j}^{k}$ is a literal, i.e., a variable or its negation. We construct an $\mathrm{UCD}_{\text {bool }}$ diagram $\mathcal{D}_{\phi}$ as follows: $\mathcal{D}_{\phi}$ contains the classes $C_{\phi}, C_{\top}$, one class $C_{i}$ for each clause $c_{i} \in \phi$, and two classes $C_{p}$ and $C_{\neg p}$ for each variable $p \in \Pi$. To describe the constraints imposed by $\mathcal{D}_{\phi}$, we provide the corresponding DL inclusion assertions, since they are more compact to write than an UCD. For every $i \in\{1, \ldots, m\}, j \in\{1,2,3\}$, and $p \in \Pi$, we have the assertions

$$
\begin{aligned}
& C_{\phi} \sqsubseteq C_{\top} \quad C_{i} \sqsubseteq C_{\top} \quad C_{l_{i}^{j}} \sqsubseteq C_{i} \\
& C_{p} \sqsubseteq C_{\top} \quad C_{\phi} \sqsubseteq C_{i} \quad C_{i} \sqsubseteq C_{\ell_{i}^{1}} \sqcup C_{\ell_{i}^{2}} \sqcup C_{\ell_{i}^{3}} \\
& C_{\neg p} \sqsubseteq C_{\top} \quad C_{\top} \sqsubseteq C_{p} \sqcup C_{\neg p} \quad C_{\neg p} \sqsubseteq \neg C_{p}
\end{aligned}
$$

Clearly, the size of $\mathcal{D}_{\phi}$ is polynomial in the size of $\phi$.

Lemma 8. A set $\phi$ of 3-clauses is satisfiable if and only if the $U C D_{\text {bool }}$ class diagram $\mathcal{D}_{\phi}$, constructed as above, is fully satisfiable.

Proof. " $\Rightarrow "$ Let $\mathcal{J} \models \phi$. Define an interpretation $\mathcal{I}=\left(\{0,1\},{ }^{\mathcal{I}}\right)$, with

$$
\begin{aligned}
& C_{\text {厂 }}^{\mathcal{I}}=\{0,1\}
\end{aligned}
$$

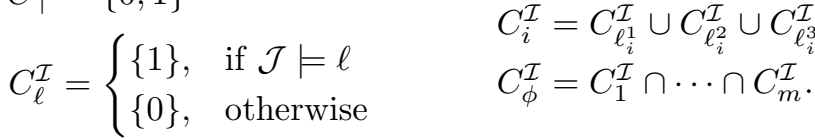

Clearly, $C^{\mathcal{I}} \neq \emptyset$ for every class $C$ representing a clause or a literal, and for $C=C_{\top}$. Moreover, as at least one literal $\ell_{i}^{j}$ in each clause is such that $\mathcal{J} \models \ell_{i}^{j}$, then $1 \in C_{i}^{\mathcal{I}}$ for every $i \in\{1, \ldots, m\}$, and therefore $1 \in C_{\phi}^{\mathcal{I}}$. It is straightforward to check that $\mathcal{I}$ satisfies $\mathcal{T}$.

" $\Leftarrow "$ Let $\mathcal{I}=\left(\Delta^{\mathcal{I}},{ }^{\mathcal{I}}\right)$ be a full model of $\mathcal{D}_{\phi}$. We construct a model $\mathcal{J}$ of $\phi$ by taking an element $o \in C_{\phi}^{\mathcal{I}}$, and setting, for every variable $p \in \Pi, \mathcal{J} \models p$ if and only if $o \in C_{p}^{\mathcal{I}}$. Let us show that $\mathcal{J} \models \phi$. Indeed, for each $i \in\{1, \ldots, m\}$, since $o \in C_{\phi}^{\mathcal{I}}$ and by the generalization $C_{\phi} \sqsubseteq C_{i}$, we have that $o \in C_{i}^{\mathcal{I}}$, and by the completeness constraint $C_{i} \sqsubseteq C_{\ell_{i}^{1}} \sqcup C_{\ell_{i}^{2}} \sqcup C_{\ell_{i}^{3}}$, there is some $j_{i} \in\{1,2,3\}$ such that $o \in C_{\ell_{i}^{j_{i}}}$. If $\ell_{i}^{j_{i}}$ is a variable, then $\mathcal{J} \models \ell_{i}^{j_{i}}$ by construction, and thus $\mathcal{J} \models c_{i}$. Otherwise, if $\ell_{i}^{j_{i}}=\neg p$ for some variable $p$, then, by the disjointness constraint $C_{\neg p} \sqsubseteq \neg C_{p}$, we have that $o \notin C_{p}^{\mathcal{I}}$. Thus, $\mathcal{J} \models \neg p$, and therefore, $\mathcal{J} \models c_{i}$. 
Theorem 9. Full satisfiability of $U C D_{\text {bool }}$ is $\mathrm{NP}$-complete

Proof. To prove the NP upper bound, we reduce full satisfiability to class satisfiability, which, for the case of $\mathrm{UCD}_{\text {bool }}$, is known to be in NP [6]. We use an encoding similar to the one used in the proof of Theorem 7 (see Fig. 8).

The NP-hardness follows from Lemma 8.

We turn now to $\mathrm{UCD}_{\text {ref }}$ class diagrams and show that full satisfiability in this case is NLOGSPACE-complete. We provide a reduction of the REACHABILITY problem on (acyclic) directed graphs, which is known to be NLOGSPACEcomplete (see e.g., [21]) to the complement of full satisfiability of $\mathrm{UCD}_{\text {ref }}$ CDs.

Let $G=(V, E, s, t)$ be an instance of REACHABILITY, where $V$ is a set of vertices, $E \subseteq V \times V$ is a set of directed edges, $s$ is the start vertex, and $t$ the terminal vertex. We construct an $\mathrm{UCD}_{\text {ref }}$ diagram $\mathcal{D}_{G}$ from $G$ as follows:

- $\mathcal{D}_{G}$ has two classes $C_{v}^{1}$ and $C_{v}^{2}$, for each vertex $v \in V \backslash\{s\}$, and one class $C_{s}$ corresponding to the start vertex $s$.

- For each edge $(u, v) \in E$ with $u \neq s$ and $v \neq s, \mathcal{D}_{G}$ contains the following constraints (again expressed as DL inclusion assertions): $C_{u}^{1} \sqsubseteq C_{v}^{1}, C_{u}^{2} \sqsubseteq C_{v}^{2}$.

- For each edge $(s, v) \in E, \mathcal{D}_{G}$ contains the following constraints: $C_{s} \sqsubseteq C_{v}^{1}$, $C_{s} \sqsubseteq C_{v}^{2}$.

- For each edge $(u, s) \in E, \mathcal{D}_{G}$ contains the following constraints: $C_{u}^{1} \sqsubseteq C_{s}$, $C_{u}^{2} \sqsubseteq C_{s}$.

- The classes $C_{t}^{1}$ and $C_{t}^{2}$ are constrained to be disjoint in $\mathcal{D}$, expressed by: $C_{t}^{1} \sqsubseteq \neg C_{t}^{2}$.

The following lemma establishes the correctness of the reduction.

Lemma 10. $t$ is reachable from $s$ in $G$ iff $\mathcal{D}_{G}$ is not fully satisfiable.

Proof. " $\Rightarrow$ " Let $\pi=v_{1}, \ldots, v_{n}$ be a path in $G$ with $v_{1}=s$ and $v_{n}=t$. We claim that the class $C_{s}$ in the constructed diagram $\mathcal{D}_{G}$ is unsatisfiable. Suppose otherwise that there is a model $\mathcal{I}$ of $\mathcal{D}_{G}$ with $o \in C_{s}^{\mathcal{I}}$, for some $o \in \Delta^{\mathcal{I}}$. From $\pi$, a number of generalization constraints hold in $\mathcal{D}_{G}$, i.e., $C_{s}^{\mathcal{I}} \subseteq C_{t}^{1^{\mathcal{I}}}$ and $C_{s}^{\mathcal{I}} \subseteq C_{t}^{2^{\mathcal{I}}}$. Thus, we obtain that $o \in\left(C_{t}^{1}\right)^{\mathcal{I}}$ and $o \in\left(C_{t}^{2}\right)^{\mathcal{I}}$, which violates the disjointness between the classes $C_{t}^{1}$ and $C_{t}^{2}$, in contradiction to $\mathcal{I}$ being a model of $\mathcal{D}_{G}$. Hence, $C_{s}$ is unsatisfiable, and therefore $\mathcal{D}_{G}$ is not fully satisfiable.

" $\Leftarrow "$ Let us consider the contrapositive. Assume that $t$ is not reachable from $s$ in $G$. We construct a full model $\mathcal{I}$ of $\mathcal{D}_{G}$. Let $\Delta^{\mathcal{I}}=\left\{d_{s}\right\} \cup \bigcup_{v \in V \backslash\{s\}}\left\{d_{v}^{1}, d_{v}^{2}\right\}$. Define inductively a sequence of interpretations as follows:

$$
\begin{aligned}
& \mathcal{I}^{0}=\left(\Delta^{\mathcal{I}}, \mathcal{I}^{0}\right), \text { such that: } C_{s}^{\mathcal{I}^{0}}=\left\{d_{s}\right\}, C_{v}^{i} \mathcal{I}^{0}=\left\{d_{v}^{i}\right\}, \forall i \in\{1,2\}, v \in V \backslash\{s\}, \\
& \mathcal{I}^{n+1}=\left(\Delta^{\mathcal{I}}, \cdot^{\mathcal{I}^{n+1}}\right) \text {, such that: } C_{s}^{\mathcal{I}^{n+1}}=C_{s}^{\mathcal{I}^{n}} \cup \bigcup_{(u, s) \in E}\left(C_{u}^{1^{\mathcal{I}^{n}}} \cup\right. \\
& \left.C_{u}^{2^{\mathcal{I}^{n}}}\right), C_{v}^{\mathcal{I}^{n+1}}=C_{v}^{\mathcal{I}^{n}} \cup \bigcup_{(u, v) \in E, u \neq s} C_{u}^{i \mathcal{I}^{n}} \cup \bigcup_{(s, v) \in E} C_{s}^{\mathcal{I}^{n}} .
\end{aligned}
$$


The definition induces a monotone operator over a complete lattice, and hence it has a fixed point. Let $\mathcal{I}$ be defined by such a fixed point. It is easy to check that $\mathcal{I}$ is such that for all $i \in\{1,2\}$, and $u, v \in V \backslash\{s\}$ the following holds:

1. For each class $C_{v}^{i}$, we have that $d_{v}^{i} \in C_{v}^{i \mathcal{I}}$.

2. $d_{s} \in C_{s}^{\mathcal{I}}$.

3. For all $d \in \Delta^{\mathcal{I}}, d \in C_{u}^{i \mathcal{I}}$ implies $d \in C_{v}^{i \mathcal{I}}$ iff $v$ is reachable from $u$ in $G$.

4. For all $d_{u}^{i} \in \Delta^{\mathcal{I}}, d_{u}^{i} \in C_{v}^{j \mathcal{I}}$ for $i \neq j$ iff $s$ is reachable from $u$ in $G$, and $v$ is reachable from $s$ in $G$.

5. $d_{s} \in C_{v}^{i \mathcal{I}}$ iff $v$ is reachable from $s$ in $G$.

From (11) and (2) we have that all classes in $\mathcal{D}_{G}$ are populated in $\mathcal{I}$. It remains to show that $\mathcal{I}$ satisfies $\mathcal{D}_{G}$. A generalization between the classes $C_{u}^{i}$ and $C_{v}^{i}$ corresponds to the edge $(u, v) \in E$. This means that $v$ is reachable from $u$ in $G$, and therefore, by (3) we have that $C_{u}^{i \mathcal{I}} \subseteq C_{v}^{i \mathcal{I}}$. A similar argument holds for generalizations involving the class $C_{s}$. Furthermore, the classes $C_{t}^{1}$ and $C_{t}^{2}$ are disjoint under $\mathcal{I}$. To show this, suppose that there is an element $d \in \Delta^{\mathcal{I}}$ such that $d \in C_{t}^{1 \mathcal{I}} \cap C_{t}^{2 \mathcal{I}}$. Then by (5),$d \neq d_{s}$, as $t$ is not reachable from $s$. Moreover, $d \neq d_{v}^{i}$ for all $i \in\{1,2\}$ and $v \in V \backslash\{s\}$. Indeed, suppose w.l.o.g. that $i=1$. Then, by (4), $d_{v}^{1} \in C_{t}^{2 \mathcal{I}}$ iff $s$ is reachable from $v$, and $t$ is reachable from $s$, which leads to a contradiction. Hence, $C_{t}^{1 \mathcal{I}} \cap C_{t}^{2 \mathcal{I}}=\emptyset$.

Theorem 11. Full-satisfiability of $U C D_{\text {ref }}$ class diagrams is NLoGSPACEcomplete.

Proof. The NLogSpace membership follows from the NLogSPACE membership of class satisfiability [6], and a reduction similar to the one used in Theorem 9. Since NLOGSPACE = CONLOGSPACE (by the Immerman-Szelepcsényi theorem; see, e.g., [21]), and as the above reduction is logspace bounded, it follows that full consistency of $\mathrm{UCD}_{\text {ref }}$ class diagrams is NLOGSPACE-hard.

\section{Conclusions}

This paper investigates the problem of full satisfiability in the context of UML class diagrams, i.e., whether there is at least one model of the diagram where each class and association is non-empty. Our results (reported in Table 1) show that the complexity of checking full satisfiability is ExPTIME-complete both in the full scenario $\left(\mathrm{UCD}_{\text {full }}\right)$ and in the case where attributes are dropped, NP-complete if we drop ISA between relationships $\left(\mathrm{UCD}_{b o o l}\right)$, and NLOGSPACEcomplete if we further drop covering over classes $\left(\mathrm{UCD}_{r e f}\right)$, thus matching the complexity of the classical class diagram satisfiability check. These complexity bounds extend the ones presented in 6] for class/schema satisfiability to full satisfiability. We show a similar result also for the problem of checking the full satisfiability of a TBox expressed in the description logic $\mathcal{A L C}$. As a future work, we intend to investigate the problem under the finite model assumption. 
Acknowledgements. This research has been partially supported by the FP7 ICT projects ACSI, contract n. 257593, and OntoRule, contract n. 231875.

\section{References}

1. Artale, A., Calvanese, D., Ibanez-Garcia, A.: Full satisfiability of UML class diagrams (extended abstract). Technical Report 127, Roskilde University Computer Science Research Reports. In: Proc. of the 2009 Int. Workshop on Logic in Databases (LID 2009) (2009)

2. Clark, T., Evans, A.S.: Foundations of the Unified Modeling Language. In: Duke, D., Evans, A. (eds.) Proc. of the 2nd Northern Formal Methods Workshop, Springer, Heidelberg (1997)

3. Evans, A., France, R., Lano, K., Rumpe, B.: Meta-modelling semantics of UML. In: Kilov, H. (ed.) Behavioural Specifications for Businesses and Systems. Kluwer Academic Publishers, Dordrecht (1999)

4. Harel, D., Rumpe, B.: Modeling languages: Syntax, semantics and all that stuff. Technical Report MCS00-16, The Weizmann Institute of Science, Rehovot, Israel (2000)

5. Berardi, D., Calvanese, D., De Giacomo, G.: Reasoning on UML class diagrams. Artificial Intelligence 168(1-2), 70-118 (2005)

6. Artale, A., Calvanese, D., Kontchakov, R., Ryzhikov, V., Zakharyaschev, M.: Reasoning over extended ER models. In: Parent, C., Schewe, K.-D., Storey, V.C., Thalheim, B. (eds.) ER 2007. LNCS, vol. 4801, pp. 277-292. Springer, Heidelberg (2007)

7. Artale, A., Calvanese, D., Kontchakov, R., Zakharyaschev, M.: The DL-Lite family and relations. J. of Artificial Intelligence Research 36, 1-69 (2009)

8. Kaneiwa, K., Satoh, K.: Consistency checking algorithms for restricted UML class diagrams. In: Dix, J., Hegner, S.J. (eds.) FoIKS 2006. LNCS, vol. 3861, pp. 219 239. Springer, Heidelberg (2006)

9. Kaneiwa, K., Satoh, K.: On the complexities of consistency checking for restricted UML class diagrams. Theoretical Computer Science 411(2), 301-323 (2010)

10. Baader, F., Calvanese, D., McGuinness, D., Nardi, D., Patel-Schneider, P.F. (eds.): The Description Logic Handbook: Theory, Implementation and Applications. Cambridge University Press, Cambridge (2003)

11. Bergamaschi, S., Sartori, C.: On taxonomic reasoning in conceptual design. ACM Trans. on Database Systems 17(3), 385-422 (1992)

12. Borgida, A.: Description logics in data management. IEEE Trans. on Knowledge and Data Engineering 7(5), 671-682 (1995)

13. Artale, A., Cesarini, F., Soda, G.: Describing database objects in a concept language environment. IEEE Trans. on Knowledge and Data Engineering 8(2), 345351 (1996)

14. Calvanese, D., Lenzerini, M., Nardi, D.: Description logics for conceptual data modeling. In: Chomicki, J., Saake, G. (eds.) Logics for Databases and Information Systems, pp. 229-264. Kluwer Academic Publishers, Dordrecht (1998)

15. Calvanese, D., Lenzerini, M., Nardi, D.: Unifying class-based representation formalisms. J. of Artificial Intelligence Research 11, 199-240 (1999)

16. Borgida, A., Brachman, R.J.: Conceptual modeling with description logics. In: [10], ch. 10, pp. 349-372 
17. Möller, R., Haarslev, V.: Description logic systems. In: [10], ch. 8, pp. 282-305

18. Buchheit, M., Donini, F.M., Schaerf, A.: Decidable reasoning in terminological knowledge representation systems. J. of Artificial Intelligence Research 1, 109-138 (1993)

19. Lenzerini, M., Nobili, P.: On the satisfiability of dependency constraints in entityrelationship schemata. Information Systems 15(4), 453-461 (1990)

20. Jarrar, M., Heymans, S.: Towards pattern-based reasoning for friendly ontology debugging. Int. J. on Artificial Intelligence Tools 17(4), 607-634 (2008)

21. Papadimitriou, C.H.: Computational Complexity. Addison Wesley Publ. Co., Reading (1994) 